\title{
Curing Behavior for Microencapsulated Curing Agents on Epoxy Resin Systems
}

\author{
Lulu Zhang ${ }^{1, \text { a }}$ Xi Wang ${ }^{1, b^{*}}$ \\ ${ }^{1}$ Xi'an University of Architecture and Technology \\ College of Materials \& Mineral Resources \\ No. 13 Yanta Road, Xi'an, Shaanxi, China, 710055

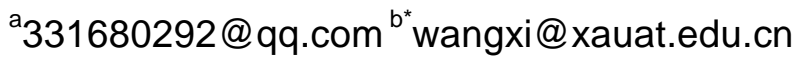

\begin{abstract}
Keywords: Curing of polymers; Curing behavior; Microencapsulation.
Abstract. Microcapsules containing a curing agent, 2-phenyl imidazole (2PZ), for a diglycidyl ether of bisphenol A (DGEBA) epoxy resin were prepared by a solid-in-oil-inwater emulsion solvent evaporation technique with poly (methyl methacrylate) (PMMA) as a polymeric wall. The former could take more than 3 months at room temperature, whereas the latter was cured after only a week. The values of the reaction order (a curing kinetic parameter) for DGEBA/2PZ and DGEBA/2PZ-PMMA microcapsules were quite close, and this showed that the curing reactions of the two samples proceeded conformably. The curing mechanism was investigated, and a two-step initiation mechanism was considered: the first was assigned to adduct formation, whereas the second was due to alkoxide-initiated polymerization..
\end{abstract}

\section{Introduction}

Epoxy resins have good thermal and dimensional stability, excellent chemical and corrosion resistance, high tensile strength and modulus, and ease of handling and processability, which ensure their widespread applications in the aerospace and electronic industries in the forms of structural adhesives, advanced composite matrices, and packaging materials ${ }^{[1,2]}$. Such excellent properties are not displayed by epoxy resins until they are cured by a certain kind of curing agent under certain conditions and are turned into networks. The properties of cured epoxy polymers depend not only on the nature of the chemical structure of the starting resins ${ }^{[3]}$ but also on the molecular structure of the curing agents and their forms in epoxy resins ${ }^{[4]}$. For some practical purposes, epoxy resins are required to be stable during storage and to be cured when they are needed; this leads to the birth of latent curing agents, including the microencapsulation of curing agents.

The solid-in-oil-in-water (S/O/W) emulsion solvent evaporation technique10 employed in this work is widely used for microencapsulation because it is rather easy to scale up, as shown in the commercially available product Lupron Depot ${ }^{[5,6]}$.

Many studies have been conducted to study the curing kinetics of epoxy resins with differential scanning calorimetry (DSC) ${ }^{[7,8]}$, isothermal DSC ${ }^{[9]}$, Raman spectrometry ${ }^{[10]}$, Fourier transform infrared (FTIR) analysis ${ }^{[1]}$, and so on. Reports on the preparation of epoxy-resin-based systems with the addition of microencapsulated curing agents have been presented many times; however, the effects of microencapsulation on the curing behavior of epoxy resin systems are expounded quite rarely.

The objective of this study was to prepare 2-phenyl imidazole (2PZ)/poly(methyl methacrylate) (PMMA) microcapsules and apply them to epoxy resin systems as latent curing agents. The curing kinetics initiated by $2 \mathrm{PZ}$ and its microencapsulated congener were analyzed with a scanning DSC technique combined with Kissinger and Crane equations. Meanwhile, the curing mechanisms were also investigated with scanning DSC combined with FTIR studies. 


\section{Experimental}

\section{Materials}

Curing agent 2PZ (98\%), used as a core material, was purchased from Energy Chemical (Shanghai, China). PMMA, used as a wall material, was supplied by Guangdong Weibo Chemical Co. (Guangzhou, Guangdong, China). Dichloromethane (DCM; a solvent) and sodium dodecyl sulfate (SDS; an emulsifier) were also supplied by J\&K Chemical Ltd. (Shanghai).

DSC studies were performed on a thermal analyzer (DSC Q100, TA, United States). The samples listed in Table I [(a) DGEBA/PMMA, (b) DGEBA/2PZ, (c) DGEBA/2PZ-PMMA microcapsules, and (d) DGEBA/ 2PZ/PMMA] were scanned at b values of 5,10 , and $20^{\circ} \mathrm{C} / \mathrm{min}$ in an $\mathrm{N}_{2}$ atmosphere. Scanning DSC stud-ies, combined with FTIR (Vector 22, Bruker, Germany; KBr), were also carried out to study the cure of the DGEBA epoxy with 2PZ and 2PZ/PMMA microcapsules (Table 1, samples 2 and 3$)$.

TABLE 1

Constituents of the samples characterized with scanning DSC

\begin{tabular}{ccccc}
\hline Sample & $\begin{array}{c}\text { DGEBA } \\
(\mathrm{g})\end{array}$ & $\begin{array}{c}\text { 2PZ } \\
(\mathrm{g})\end{array}$ & $\begin{array}{c}\text { PMMA } \\
\text { Mircospheres(g } \\
)\end{array}$ & $\begin{array}{c}\text { 2PZ/PMMA } \\
\text { Microcapsules }\end{array}$ \\
\hline 1 & 100 & 0 & 15 & 0 \\
2 & 100 & 2 & 0 & 0 \\
3 & 100 & 0 & 0 & 20 \\
4 & 100 & 2 & 15 & 0 \\
\hline
\end{tabular}

\section{Results and Discussion}

Characterization of the prepared microparticles The process of microencapsulation was performed because of the higher drug loading ratio and suitable particle size. The morphologies of the 2PZ/PMMA microcapsules and PMMA microspheres were characterized with SEM, as shown in Figure 1. Regular spheres of the 2PZ/PMMA microcapsules and PMMA microspheres with a well-proportioned size of about $10 \mathrm{~mm}$ were observed, and the PMMA microspheres were slightly smaller than the 2PZ/ PMMA microcapsules. The surface of the microparticles was coarse and porous under a high magnification $(15,000 \times)$. Besides, when the 2PZ/PMMA microcapsules were pestled and washed with glycol, a core-wall structure with thick walls and irregular small cores could be found with SEM.

Effect of microencapsulation on the curing mechanism The curing mechanisms of samples $\mathrm{b}$ and $\mathrm{c}$ were studied with a scanning DSC technique. The curing behaviors of DGEBA/2PZ and DGEBA/2PZ-PMMA microcapsules (Table 1, samples 2 and 3) undergoing five different heating histories were studied with scanning DSC and FTIR. Figure 1 shows the DSC curves of sample b undergoing five different heating histories. In Figure 7, two exothermal peaks can be distinguished in curves 1 and 2, and the first exothermal peak becomes much flatter, but the second one shows no obvious change after the heating of the sample at $110^{\circ} \mathrm{C}$ for $1.5 \mathrm{~h}$. However, after the heating of the sample at 130 and $160^{\circ} \mathrm{C}$ for $1.5 \mathrm{~h}$, respectively [Fig. 1(3,4)], the first peak disappears, and the second one becomes more and more feeble and finally vanishes [Fig. 2(5)]. Figure 8 displays scanning DSC curves of sample c under five different heating histories, and the same trend revealed in Figure 1 is exhibited. Meanwhile, the glass-transition temperature ( $\mathrm{Tg}$ ) values of completely cured samples $b$ and c, determined by DSC curves [Figs.1(5) and 2(5)], were 165.20 and $142.41^{\circ} \mathrm{C}$. Tg of sample b, which was a bit higher than that reported by Ooi et al.20 (the highest was $155^{\circ} \mathrm{C}$ ), was nearly $20^{\circ} \mathrm{C}$ higher than that of c, probably because of the addition of PMMA, which can be used to increase the toughness of cured epoxy polymers because of its flexible molecular chain. 


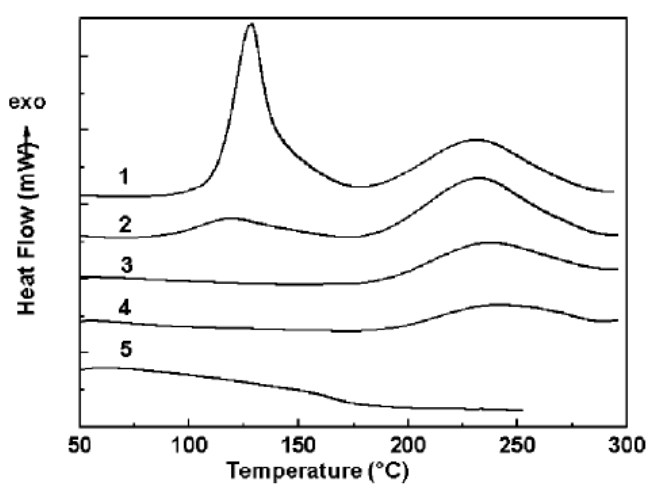

Figure 1 Scanning DSC curves of sample b undergoing five different heating histories defined at $5^{\circ} \mathrm{C} / \mathrm{min}$ under an $\mathrm{N}_{2}$ flow.

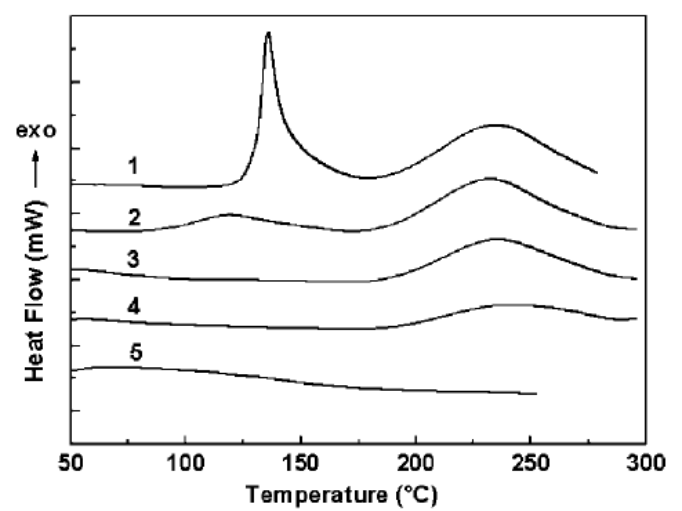

Figure 2 Scanning DSC curves of sample c undergoing five different heating histories defined at $5^{\circ} \mathrm{C} / \mathrm{min}$ under an $\mathrm{N}_{2}$ flow.

Curing mechanism of DGEBA/2PZ is proposed that is based on the work of Ooi et al. and Barton and Shepherd, and a sketch of the curing mechanism of DGEBA/2PZ is given in Schemes 1 and 2. The formation of the 1:1 adduct is generated through an attack on the epoxy-functional group of DGEBA by the more basic pyridine-type nitrogen of $2 \mathrm{PZ}$. Then, the newly generated pyridine-type nitrogen attacks another epoxy group to produce the 1: 2 adduct (Scheme 1). In the second step, these generated adducts are assumed to act as the initiators for the polymerization of DGEBA by an etherification reaction in which the reactive alkoxide anion is the propagating species (Scheme 2). Ooi et al. also reported that two exothermal peaks could be observed in 2PZ curing systems; the first peak was assigned to adduct formation, whereas the second one was due to alkoxide-initiated polymerization. The first peaks in Figures 1(2) and 2(2), much flatter than those in Figures 1(1) and $2(1)$, are due to adducts generated by the consumption of $2 \mathrm{PZ}$ after heating. When $2 \mathrm{PZ}$ is exhausted, the first reaction step (Scheme 1) is over, and the first peaks attributable to the first step disappear in Figures 1(3) and 2(3). As the samples are heated subsequently, the second reaction step (Scheme 2) takes place, and it results in the second peak area decreasing and vanishing at last, as shown in Figures 1(3-5) and 2(3-5). According to the mechanism mentioned previously, $2 \mathrm{PZ}$ is not involved in the second curing reaction step of a DGEBA epoxy resin.

\section{Conclusions}

The solvent evaporation method was applied to prepare 2PZ/PMMA microcapsules, in which the concentration of $2 \mathrm{PZ}$ was $10 \mathrm{wt} \%$, as determined by TGA. Regular spherical microcapsules and PMMA microspheres with a well-proportioned size of about $10 \mathrm{~mm}$ were characterized with SEM. DGEBA/2PZ was cured after 7 days, whereas the DGEBA/2PZ-PMMA microcapsule system was still fluid even after more than 3 months at room temperature. The $n$ values of the employed systems were quite close, and this shows that the curing reactions of the two samples proceeded conformably and that microencapsulation may not influence curing reactions of DGEBA. 
The proposed curing mechanism for the $2 \mathrm{PZ}$ curing epoxy resin can explain the phenomenon perfectly. Data obtained from FTIR, combined with scanning DSC studies, for DGEBA/2PZ and DGEBA/2PZ-PMMA microcapsules undergoing five different heating histories showed that no obvious mechanical change occurred when the curing agents were replaced by 2PZ/PMMA microcapsules.

\section{Acknowledgement}

This work was financially supported by Xi'an University of Architecture and Technology（Grand No.DB09071).

\section{References}

[1] Lubin, G, Handbook of Composites, Van Nostrand Reinhold, New York, 1982.

[2] Chen, Y.; Chiu, W.; Lin, K. Kinetics study of imidazole-cured epoxy-phenol resins. J Polym Sci Part A: Polym Chem 1999, 37, 3233-3242.

[3] Xu, K.; Chen, M.; Zhang, K.; Hu, Synthesis and characterization of novel epoxy resin bearing naphthyl and limonene moieties, and its cured polymer. J. Polymer 2004, 45, 1133.

[4] G. T. Vladisavljevic, R. A. Williams. Recent developments in manufacturing emulsions and particulate products using membranes. J. Advan in Coll and Interface Sci 2005,113, 1-20

[5] K. Hong, K. Nakayama, S. Park. Effect of protective colloids on preparation of poly (1-lactide) /poly (butylene-succinate) microcapsules. J. Eur Polym, 2002, 38:305-311.

[6] C.C. Su, E.M. Woo. Cure kinetics and morphology of amine-cured tetraglycidyl-4,4'-diaminodiphenylmethane epoxy blends with poly (ether imide). J. Polymer, 1995, 36: 2883-2894.

[7] A. Loxley, B. Vincent. Preparation of Poly (methylmethacrylate) Microcapsules with Liquid Cores. J. Coll Interface Sci, 1998, 208:4962.

[8] J. M. Barton, I. Hamerton, B. J. Howlin, J. R. Jones, S. Liu. Studies of temperature and time-dependent network formation in commercial epoxy resins using modified imidazole curing agents. J. Polym Intern. 1996, 41:159-168.

[9] Rocks, J.; Rintoul, L.; Vohwinkel, F. The kinetics and mechanism of cure of an amino-glycidyl epoxy resin by a co-anhydride as studied by FT-Raman spectroscopy. J. Polymer 2004, 45, 6799.

[10] Scherzer, T.; Decker, U. The effect of temperature on the kinetics of diacrylate photopolymerizations studied by Real-time FTIR spectroscopy. J. Polymer 2000, 41, 7681. 